Proceedings of the 16th Czech and Slovak Conference on Magnetism, Košice, Slovakia, June 13-17, 2016

\title{
The Influence of Temperature on Unidirectional Effect in Domain Wall Propagation
}

\author{
J. ONUfER ${ }^{a, *}$, J. ZIMAN $^{a}$, M. REZnIČÁK ${ }^{a}$ AND S. KARDOŠ ${ }^{b}$ \\ ${ }^{a}$ Department of Physics, Faculty of Electrical Engineering and Informatics, Technical University of Košice, \\ Park Komenského 2, 04200 Košice, Slovakia \\ ${ }^{b}$ Department of Technologies in Electronics, Faculty of Electrical Engineering and Informatics, \\ Technical University of Košice, Park Komenského 2, 04200 Košice, Slovakia
}

\begin{abstract}
It has been reported recently that domain wall mobility in Fe-based amorphous glass-coated microwire can be significantly different in the cases when magnetization reversal caused by domain wall motion results in different orientation of magnetization. This behaviour has been called unidirectional effect. The effect of temperature on domain wall velocity vs. axial magnetic field dependences was studied for glass-coated $\mathrm{Fe}_{77.5} \mathrm{Si}_{7.5} \mathrm{~B}_{15}$ microwire samples with strong unidirectional effect. Unidirectional effect was observed for the whole temperature interval from $100 \mathrm{~K}$ up to room temperature. Analysis of the results obtained indicates that the model of a solid domain wall does not explain the observed $v(H)$ dependences. It seems very probable that besides standard damping mechanisms also changes in structure (shape) of the domain wall as a function of velocity (magnetic field) should be considered. Understanding of this mechanism could also provide interpretation of unidirectional effect.
\end{abstract}

DOI: 10.12693/APhysPolA.131.723

PACS/topics: 75.30.Gw, 75.50.Kj, 75.60.-d, 75.60.Ch, 75.60.Jk

\section{Introduction}

Bistable glass-coated microwires are interesting materials for the study of fundamental physics problems connected with the magnetic domain wall (DW) dynamics. The combination of soft magnetic properties, high magnetic reversal speed, small geometrical dimensions and low production costs makes them also suitable for many technical applications $[1,2]$.

It is known that the domain structure of microwires with positive magnetostriction consists of a major axial domain in the metal core, which is surrounded by many antiparallel radial domains in the shell [3]. Closure domains with magnetizations opposing that of the major domain and decreasing stray fields are created at both ends of the wire. This unique domain structure is the result of two dominant anisotropies. The first is the magnetoelastic anisotropy and the second is the shape anisotropy.

Magnetic reversal usually starts with depinning of a single domain wall from the wire end and continues with its propagation along the entire wire as a large Barkhausen jump. After this reversal the magnetization in the axial domain can have only one of two states $+M_{S}$ or $-M_{S}$. This is called magnetic bistability, one of the characteristic features of highly magnetostrictive microwires.

The existence of a very interesting phenomenon, observed in glass coated $\mathrm{Fe}_{77.5} \mathrm{Si}_{7.5} \mathrm{~B}_{15}$ microwire, has been recently reported in [4]. For a given wire region (i.e. part

*corresponding author; e-mail: jozef.onufer@tuke.sk of the wire between two pick-up coils in the Sixtus-Tonks experiment) two different velocities can be measured. If the wall moves from end $\mathrm{A}$ to end $\mathrm{B}$, two types of wall (head-to-head or tail-to-tail) and corresponding velocities can be distinguished (Fig. 1).

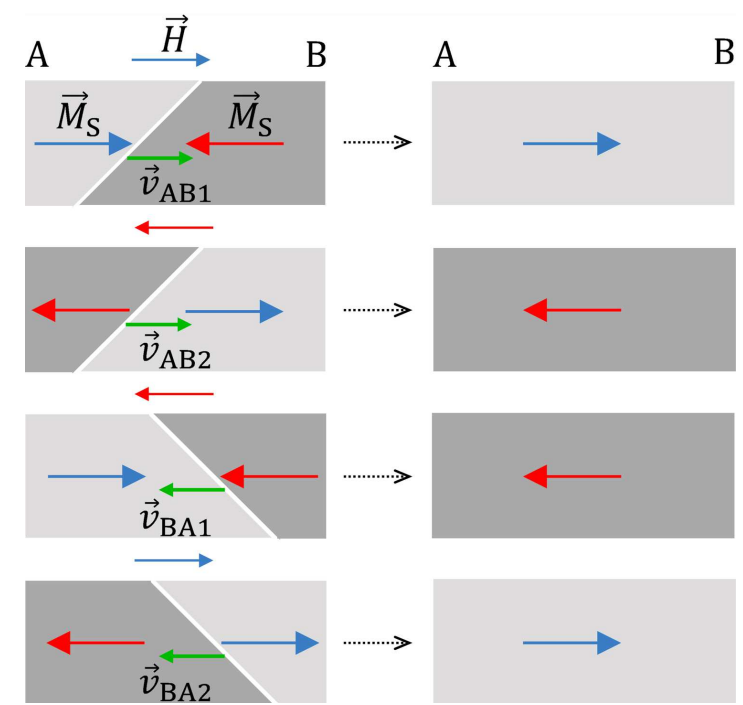

Fig. 1. Four possible magnetization reversal processes due to propagation of a single domain wall along a bistable microwire [3].

The letters A, B indicate the direction of DW motion, index 1 means the head-to-head and index 2 the tail-totail type of wall respectively. In the experimental arrangement $[4,5]$ the DW can move only from left to right (from end $\mathrm{A}$ to end $\mathrm{B}$ ) with velocity $v_{A B}$. If the wire is reversed, the DW moves from end $\mathrm{B}$ to end $\mathrm{A}$ with velocity $v_{B A}$. The so-called unidirectional effect means that 
$v_{A B 1}(H) \approx v_{B A 2}(H) \neq v_{A B 2}(H) \approx v_{B A 1}(H)$, i.e. the process of magnetization reversal is different when the sample is magnetized with one orientation of magnetization compared with the opposite one [4]. Interpretation of this phenomenon is not clear. The aim of this work was to collect more information about this phenomenon and possibly contribute to its interpretation.

It has already been shown that domain wall mobility is influenced by applied tensile stress and by circular magnetic field created by dc electric current flowing through the microwire for both $v(H)$ dependences [5].

\section{Experimental}

Amorphous glass-coated $\mathrm{Fe}_{77.5} \mathrm{Si}_{7.5} \mathrm{~B}_{15}$ microwire produced by the Taylor-Ulitovsky method was used in the experiment. The diameter of the metallic nucleus was about $15 \mu \mathrm{m}$, thickness of glass coating was about $7.5 \mu \mathrm{m}$ and length of the sample was $12 \mathrm{~cm}$.

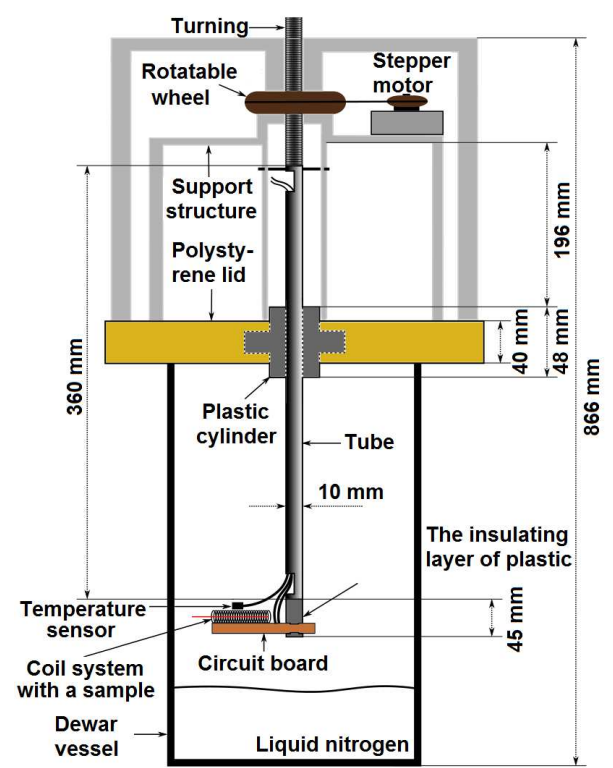

Fig. 2. Experimental setup.

The experimental setup used to measure DW velocity versus driving field dependences at different temperatures is depicted in Fig. 2. The system of coils, their dimensions and their function as well as the procedures for measurement of DW velocity (using the Sixtus-Tonks method) for a given value of the driving magnetic field have been described in [4]. To avoid possible influence of unsteady magnetizing field [6] only signals from pick up coils for which the field already reached steady value were taken for further processing in our experiment (see inset (a) in Fig. 3).

The system of coils with the studied sample is inserted into a Dewar vessel which is filled with liquid nitrogen. To change or stabilize temperature the sample holder can be moved up or down above the liquid nitrogen surface using a computer-controlled stepper motor. The temperature is measured with a thermometer based on a Pt100 resistor. The experimental setup allows the $v(H)$ dependences to be measured at constant temperature in the interval from $100 \mathrm{~K}$ up to room temperature.

\section{Results and discussion}

Samples with strong unidirectional effect were chosen for our measurements. The presence of unidirectional effect was checked by measurement of $v(H)$ dependences for all four velocities schematically shown in Fig. 1 . In the text below only one pair of $v(H)\left(v_{A B 1}(H)\right.$ and $\left.v_{A B 2}(H)\right)$ dependences will be presented and the wall with higher velocity will be called "fast" and the wall with lower velocity will be called "slow".

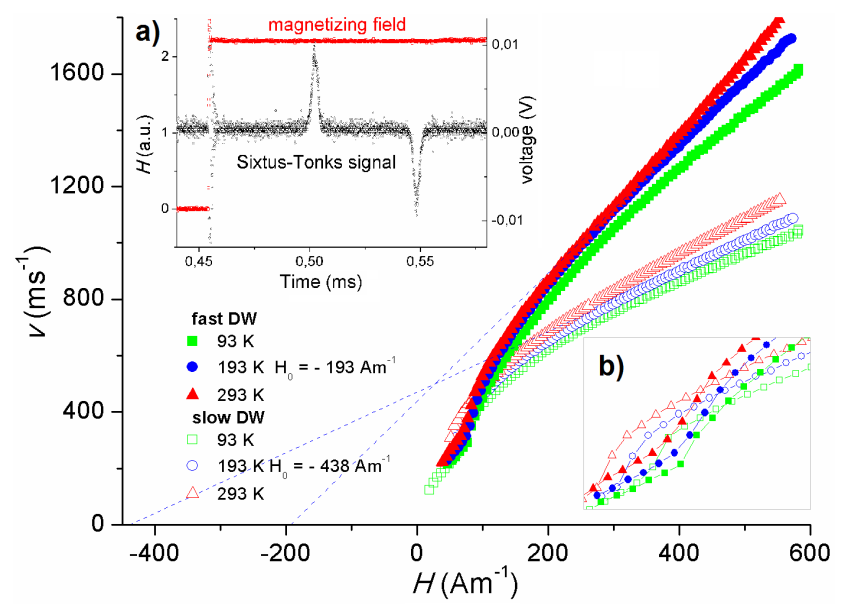

Fig. 3. DW velocity versus axial field dependences for fast and slow DW at three temperatures. Determination of $H_{0}$ from $v(H)$ dependences is shown for temperature $193 \mathrm{~K}$. Inset (b) shows detail of low field region. Inset (a) shows record from two-channel oscilloscope (one channel - magnetizing field, another channel — signal from pick up coils).

Velocity vs. driving magnetic field dependences for slow $(\square, \circ, \Delta)$ and fast $(\boldsymbol{\bullet}, \bullet, \boldsymbol{\Delta})$ DW at three temperatures $(93,193,293 \mathrm{~K})$ are shown in Fig. 3. The relative difference in velocity of fast and slow DW as a function of the driving field at the same temperatures is depicted in Fig. 4. For both types of dependences and at all temperatures different behaviour in low and high fields can be observed. It is very probable that in the field interval of $50-100 \mathrm{~A} / \mathrm{m}$ some transformation in the wall structure (or its shape) takes place. It is interesting that this transformation appears in a lower field for the slow DW (see inset in Fig. 3). Since this transformation causes a relatively abrupt increase in velocity, the quantity $\left(v_{\text {fast }}-v_{\text {slow }}\right) / v_{\text {slow }}$ in Fig. 4 can be negative in a narrow field interval.

Now we will discuss $v(H)$ dependences in higher fields. Usually a linear function of the type $v=S\left(H-H_{0}\right)$, where $S$ is wall mobility and $H_{0}$ is the so-called critical propagating field, is used in this field region. Temperature dependences of $S$ and $H_{0}$ are plotted in Fig. 5 . As already reported in [7], the field $H_{0}$ can be negative. In our samples too $H_{0}$ is negative and not the same for 


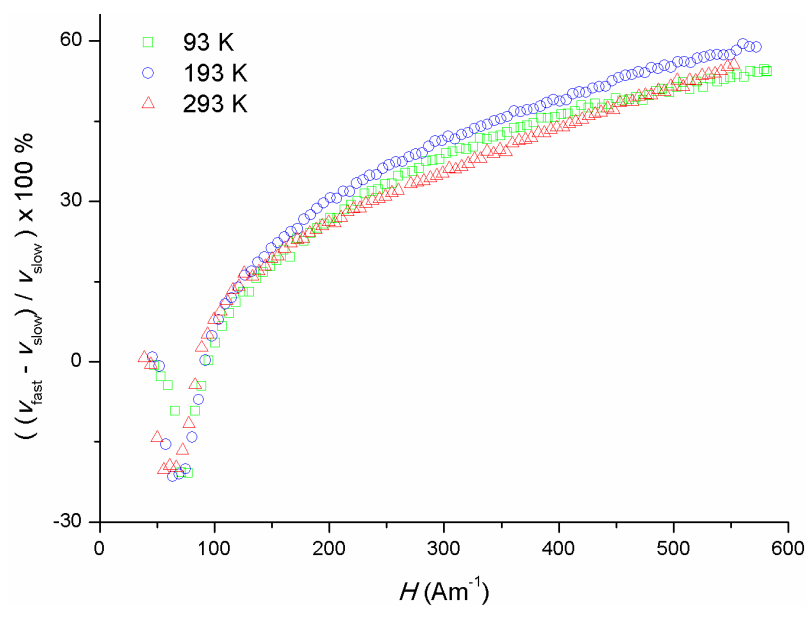

Fig. 4. Relative difference in velocities of fast and slow DW as a function of driving magnetic field at the three temperatures.

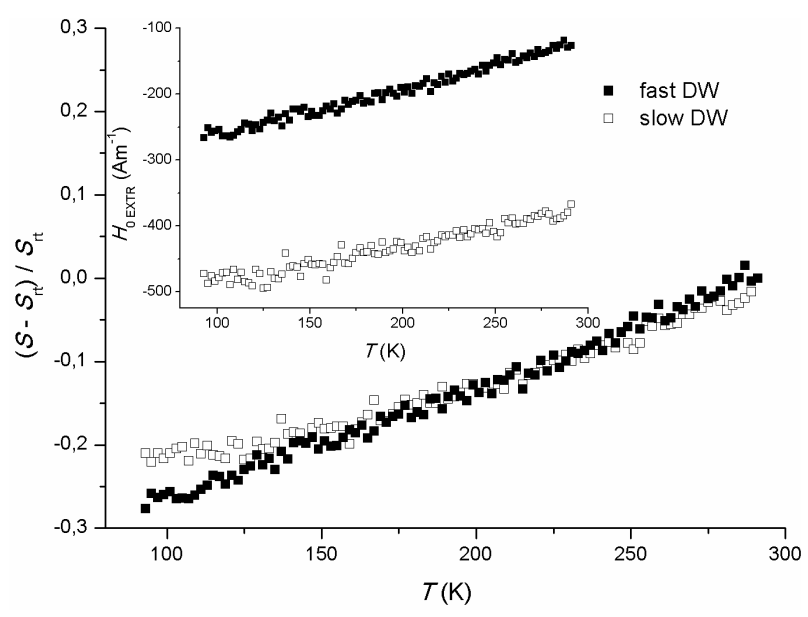

Fig. 5. Relative change in DW mobility as a function of temperature for fast and slow DWs. $S_{r t}$ is mobility at room temperature. Inset shows temperature dependence of the field $H_{0}$ for fast and slow DWs.

slow and fast DWs. The magnitude of $H_{0}$ is surprisingly high. It increases with decreasing temperature, and for the slow DW at a temperature of $100 \mathrm{~K} H_{0}=-480 \mathrm{~A} / \mathrm{m}$. The sign, magnitude and different values of $H_{0}$ for slow and fast DWs can hardly be interpreted using the model of a solid DW (fixed shape/structure).

As can be seen in Figs. 3 and 5 DW mobility decreases with decrease of temperature for both types of DW.

On the other hand, the relative difference in velocity in Fig. 4 for the high field region is only slightly influenced by temperature. It was shown in [4] that $v(H)$ dependences (and also unidirectional effect) can vary in different pieces of wire. Unidirectional effect can even change if average velocity is measured in different parts of the same piece of microwire [4]. It is generally accepted that defects in the microwire can influence DW velocity [8] but they do not explain changes in unidirectional effect. We repeated the measurement presented in Figs. 2-5 in four pieces of microwire with "strong" unidirectional ef- fect. The observed qualitative behaviour was the same as for the sample presented in Figs. 2-5 but for instance the magnitude of relative change in mobility at minimum temperature $(100 \mathrm{~K})$ is in the interval $(0.2,0.31)$. This interval seems to be too wide for the model of solid DW and three damping mechanisms (eddy currents, spin relaxation, structural relaxation) are usually used for interpretation of temperature dependence of DW dynamic parameters [7]. Based on these results it seems very probable that model of a solid DW cannot correctly explain the measured $v(H)$ dependences in Fig. 3. Besides standard damping mechanisms, changes in structure (shape) of the DW [9] as a function of velocity (field) should also be considered.

\section{Conclusions}

The effects of temperature on DW velocity vs. axial magnetic field dependences were studied in glass-coated microwire samples with strong unidirectional effect. Unidirectional effect was observed in the whole temperature interval from $100 \mathrm{~K}$ up to room temperature. The model of a solid DW does not explain the observed $v(H)$ dependences. Besides standard damping mechanisms, changes in structure (shape) of the DW as a function of velocity (field) should probably also be considered. Understanding of this mechanism could also provide interpretation of the unidirectional effect.

\section{Acknowledgments}

This research was supported by VEGA grant No. 1/0413/15 of the Scientific Grant Agency of the Ministry for Education of the Slovak Republic and also by the project "Centre of Excellence for Integrated Research \& Exploitation of Advanced Materials and Technologies in Automotive Electronics" ITMS 26220120055.

\section{References}

[1] V. Zhukova, M. Ipatov, A. Zhukov, Sensors 9, 9216 (2009).

[2] D. Praslička, J. Blažek, M. Šmelko, J. Hudak, A. Čverha, I. Mikita, R. Varga, A. Zhukov, IEEE Trans. Magn. 49, 1 (2013).

[3] H. Chiriac, T.A. Ovari, G. Pop, Phys. Rev. B 52, 10104 (1995).

[4] J. Onufer, J. Ziman, M. Kladivová, J. Magn. Magn. Mater. 396, 313 (2015).

[5] J. Onufer, J. Ziman, M. Kladivová, J. Electr. Eng. 66, 108 (2015).

[6] M. Ipatov, V. Zhukova, A.K. Zvezdin, A. Zhukov, J. Appl. Phys. 106, 103902 (2009).

[7] R. Varga, G. Infante, K. Richter, M. Vázquez, Phys. Status Solidi A 208, 509 (2011).

[8] V. Rodionova, V. Zhukova, M. Ilyn, M. Ipatov, N. Perov, A. Zhukov, Physica B 407, 1446 (2012).

[9] S.A. Gudoshnikov, Yu.B. Grebenshchikov, B.Ya. Ljubimov, P.S. Palvanov, N.A.Usov, M. Ipatov, A. Zhukov, J. Gonzalez, Phys. Status Solidi A 206, 613 (2009). 